\title{
INVARIANT IMBEDDING AND VARIATIONAL PRINCIPLES IN TRANSPORT THEORY
}

\author{
BY RICHARD BELLMAN, ${ }^{1}$ ROBERT KALABA ${ }^{1}$ AND G. MILTON WING ${ }^{2}$ \\ Communicated by R. P. Boas, March 24, 1961
}

1. Introduction. In a series of papers we have investigated various aspects of the determination of internal, reflected, and transmitted fluxes using invariance principles. A survey of these results, with reference to the classical work of Ambarzumian and Chandrasekhar, and to more recent work by the authors and others, will be found in our paper [1].

Our objective in this paper is to show that we can find a single variational problem which yields the linear equations of conventional transport theory when treated by means of the calculus of variations, and which yields the nonlinear equations of invariant imbedding when approached by means of the functional equation technique of dynamic programming [2].

2. The transport model. Consider a steady-state transport process, involving absorption, fission and scattering, taking place in a onedimensional medium, a rod of length $T$. For the sake of simplicity, we shall suppose that the rod is homogeneous and isotropic.

To treat internal flux, we introduce the vector $u(y)$ $=\left(u_{1}(y), \cdots, u_{N}(y)\right)$, where $u_{i}(y)$ denotes the expected flux of particles of type $i$ passing the internal point $y$ to the right per second. Similarly, the flow to the left is represented by the vector $v(y)$.

To treat reflected flux, we introduce the matrix $R(T)=\left(r_{i j}(T)\right)$, where $r_{i j}(T)$ is the expected reflected flux in state $i$ due to a unit incident flux in state $j$ incident at $T$.

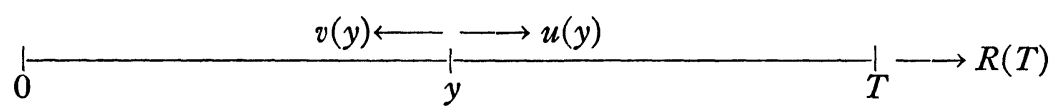

The basic interactions are expressed in terms of the matrices $A=\left(a_{i j}\right)$ and $B=\left(b_{i j}\right)$ where

1 The RAND Corporation, Santa Monica, California.

${ }^{2}$ Sandia Corporation, Albuquerque, New Mexico. 
(1) $a_{i j} \Delta+o(\Delta)=$ the expected incremental number of particles in state $i$ forward-scattered from a rod of length $\Delta$ per incident particle in state $j$,

$b_{i j} \Delta+o(\Delta)=$ the expected incremental number of particles in state $i$ back-scattered from a rod of length $\Delta$ per incident particle in state $j$.

Our present variational method forces us to assume that $B$ is symmetric and positive definite. Subsequently, we shall present a more sophisticated approach relaxing this condition which holds only in very special physical models.

The notation here follows that used in [1].

3. An extremal problem. Let us pose the problem of finding vector functions $u$ and $v$ which extremize the quadratic functional

$$
J(u, v)=\int_{0}^{r}\left\{(\dot{u}, v)-\frac{1}{2}[(B u, u)+(B v, v)+2(A u, v)]\right\} d y,
$$

where $u$ and $v$ are subject to the boundary conditions

$$
u(0)=0, \quad v(T)=c,
$$

and the dot denotes differentiation with respect to $y$.

4. Euler equations and internal flux. The Euler equations for the extremal problem of $\S 3$ are readily seen to be

$$
\begin{aligned}
\text { (a) } \dot{u} & =A u+B v, \\
\text { (b) }-\dot{v} & =B u+A^{\prime} v,
\end{aligned}
$$

with the two-point boundary conditions of (3.2). These are precisely the equations for the internal fluxes given in [1], where $A=A^{\prime}$. This, of course, is no accident, since the quadratic functional $J$ was constructed, along the lines of Hamilton-Jacobi theory, to yield these variational equations.

5. Functional equations and reflected fluxes. Returning to $J(u, v)$, let us adjoin the differential equation of (4.1a). Then the quadratic functional assumes the simpler form

$$
J(u, v)=\frac{1}{2} \int_{0}^{T}\{(B v, v)-(B u, u)\} d t .
$$

Denote by $f(c, T)$ the maximum of $J(u, v)$ over all $u$ where $u$ and $v$ are related by (4.1b), and subject to the boundary conditions of (3.2). The principle of optimality [2] yields the relation 


$$
\begin{aligned}
f(c, T+\Delta)=\max _{w}\left[\frac{1}{2}((B c, c)\right. & -(B w, w)) \Delta \\
& \left.+f\left(c+\left(B w+A^{\prime} c\right) \Delta, T\right)\right]+o(\Delta),
\end{aligned}
$$

where $w=w(T)$.

Passing to the limit as $\Delta \rightarrow 0$, we obtain the nonlinear partial differential equation

$$
\frac{\partial f}{\partial T}=\max _{\omega}\left[\frac{1}{2}((B c, c)-(B w, w))+\left(B w+A^{\prime} c, \operatorname{grad} f\right)\right] .
$$

Since the maximization can be carried out explicitly, this relation can be written

$$
\begin{aligned}
\frac{\partial f}{\partial T}=\frac{1}{2}\left[(B c, c)+(A \operatorname{grad} f, c)+\left(A^{\prime} c, \operatorname{grad} f\right)\right. & \\
& +(B \operatorname{grad} f, \operatorname{grad} f)],
\end{aligned}
$$

a Hamilton-Jacobi equation, with the boundary conditions $f(c, 0)=0$.

The linearity of the equations of (4.1) permits us to conclude that $f(c, T)$ is a quadratic form in $c$,

$$
f(c, T)=\frac{1}{2}(Z(T) c, c) .
$$

Substituting in (4), we obtain, after some simple algebra, the ordinary differential equation

$$
\frac{d Z}{d T}=B+A Z+Z A^{\prime}+Z B Z, \quad Z(0)=0 .
$$

This is an equation of Riccatian form, familiar to us in the theory of control processes [3] and in quasilinearization theory [4].

We recognize this equation to be identical, when $A=A^{\prime}$, with the equation derived for $R(T)$ by use of invariance principles, see [1]. Hence we have the interesting and important facts that

$$
\begin{aligned}
f(c, T) & =\frac{1}{2}(R(T) c, c), \\
u(T) & =R(T) c=\operatorname{grad} f .
\end{aligned}
$$

6. An alternative approach. Peter Lax has pointed out to us that we may evaluate $f(c, T)$ using the Euler equations above. We write, assuming $A=A^{\prime}$, 


$$
\begin{aligned}
f(c, T) & =\frac{1}{2} \int_{0}^{T}[(\dot{u}-A u, v)+(\dot{v}+A v, u)] d t \\
& =\frac{1}{2} \int_{0}^{T}[(\dot{u}, v)+(\dot{v}, u)] d t \\
& =\left.\frac{1}{2}(u, v)\right|_{0} ^{T} \\
& =\frac{1}{2}(u(T), v(T)) \\
& =\frac{1}{2}(R(T) c, c) .
\end{aligned}
$$

7. Discussion. There are three levels of significance to the preceding results. At the conceptual level, they provide a unified approach to the treatment of internal and external fluxes along classical and modern lines. At the analytic and computational levels they enable us to obtain various inequalities for the reflected fluxes and to apply Rayleigh-Ritz techniques to the determination of fluxes and critical lengths.

At the moment we have been proceeding formally, and under strong restrictions on the properties of the matrices $A$ and $B$. These restrictions may be somewhat relaxed. Our methods carry through directly in some problems when the matrices are symmetrizable [5]. The more general cases involving nonsymmetric matrices may be attacked by min-max methods. Such problems, together with similar considerations for nonlinear equations, will be presented in future papers, together with the rigorous aspects.

\section{REFERENCES}

1. R. Bellman, R. Kalaba, and G. M. Wing, Invariant imbedding and mathematical physics. I, Particle processes, J. Math. Phys. vol. 1 (1960) pp. 280-308.

2. R. Bellman, Dynamic programming, Princeton, N. J., Princeton University Press, 1957.

3. —, Adaptive control processes: A guided tour, Princeton, N. J., Princeton University Press, 1961.

4. R. Kalaba, On nonlinear differential equations, the maximum operation, and monotone convergence, J. Math. Mech. vol. 8 (1959) pp. 519-574 (especially pp. 525527).

5. R. Bellman, Introduction to matrix analysis, New York, McGraw-Hill Book Co., Inc., 1960, p. 67.

RAND CORPORATION AND

SANDia CoRporation 